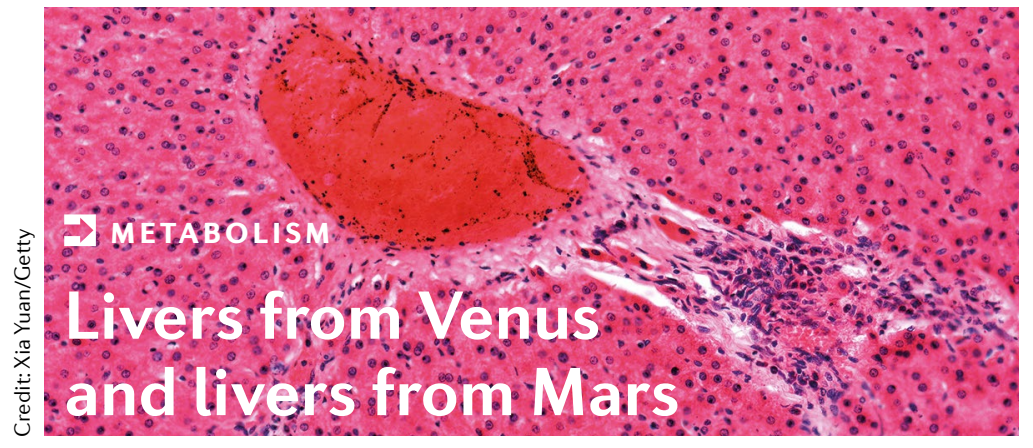

New research has reported sex differences in how the livers of mice respond to short-term fasting. Although data exist showing that the liver is a sexually dimorphic organ, the reasons for this dimorphism were unclear until now.

In animals, a mutual interaction exists between liver activity and reproductive function. "In oviparous species, the liver is essential for ovarian functions because it provides the proteins for the maturation of the egg," explain Adriana Maggi and Sara Della Torre. This feature has relevant functional consequences because it ensures that reproduction does not occur when food is scarce.
"In mammals the liver can recognize when the female is cycling, pregnant or lactating and adjust its energy production accordingly," adds Maggi.

The team were interested in investigating the mechanisms controlling the sexual dimorphism of the mammalian liver as males are not affected by changes in reproductive status. To do this, Adriana Maggi, Sara Della Torre and colleagues compared the metabolome and transcriptome of livers from adult mice in a condition of short-term fasting. "The mild starvation protocol was necessary to limit activation of the hepatic

oestrogen receptor by dietary amino acids, a protocol that we adopted to maximize the sex differences due to physiological sex hormones," explains Della Torre.

The authors report that in response to short-term fasting, the livers of male mice stop producing energy storage molecules, while the livers of female mice make use of all available resources, including amino acids, to increase the available pool of lipids. Intriguingly, the authors found that the hepatic oestrogen receptor- $\alpha$ is integral to the adoption and maintenance of this sex-specific response to short-term fasting.

"The finding that in female mice the hepatic oestrogen receptor- $\alpha$ has a key role in the control of energy metabolism points to the possibility of selectively targeting this receptor for a novel generation of therapies for the post-menopause," concludes Maggi.

Alan Morris

the post-

menopause
ORIGINAL ARTICLE Della Torre, S. et al. Short-term fasting reveals amino acid metabolism as a major sex-discriminating factor in the liver. Cell Metab. https://doi.org/10.1016/j.cmet.2018.05.021 (2018)

\title{
Microbiota alters behaviour
}

Type 2 diabetes mellitus and obesity are associated with increased rates of depression, anxiety and other mood disorders. Furthermore, a diet that contains large quantities of fat can alter the gut microbiota by reducing bacterial diversity and modifying the microbial composition. In addition, research suggests that changes to the overall bacterial composition of the gut microbiota can induce systemic insulin resistance, which is notable for neuropsychiatric disorders as the brain is sensitive to fluctuations in serum levels of insulin.

What remained unclear, however, was whether dietary changes and changes to the gut microbiota by antibiotics affected mood and behaviour and, more importantly, whether these changes to behaviour were mediated by changes in the microbiome in the gut. Now, C. Ronald Kahn and colleagues have reported that the modification of gut microbiota due to high-fat diet (HFD) feeding results in multiple behavioural abnormalities indicative of anxiety and depression in mice.

To assess whether a link exists between the gut microbiota and brain function in the context of obesity, the authors fed mice a HFD with or without the addition of antibiotics that modify the microbiome independent of diet. Khan and colleagues then assessed the behaviour of the mice and tested whether changes in behaviour could be transferred to germ-free mice via the transfer of gut microbiota.

"We showed that a HFD induced changes in behaviour consistent with increased anxiety and depression," explains Kahn. "These changes were reversed by antibiotic treatment that further modified the microbiome and the behaviours were transferable to germ-free mice." The investigators also found that the behavioural changes in the mice were associated with changes in insulin signalling in the brain, as well as changes in neurotransmitters and

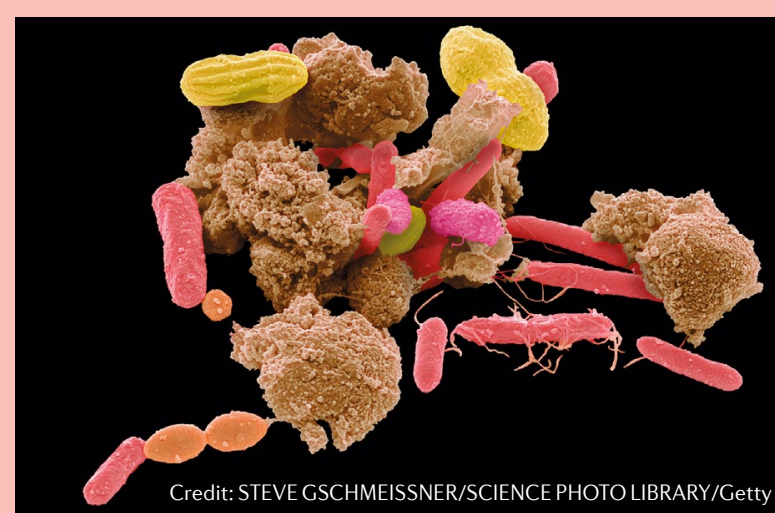

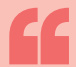

the modi-

fication of gut

microbiota

due to high-

fat diet (HFD)

feeding results

in multiple

behavioural

abnormalities metabolites in the blood that can affect brain function.

"Our current goal is to identify the metabolites that are produced by the microbiome that cause these behavioural changes," concludes Khan. "If [we can achieve this], we can design new targets for treatment of anxiety and depressive disorders."

Alan Morris

ORIGINAL ARTICLE Soto, M. et al. Gut microbiota modulate neurobehavior through changes in brain insulin sensitivity and metabolism. Mol. Psychiatry https://doi.org/10.1038/s41380-018-0086-5 (2018) potentially use these metabolites to 